\title{
The Challenge of Modelling Galactic Disks
}

\author{
Andreas Burkert ${ }^{1}$ \\ ${ }^{1}$ University Observatory, University of Munich, \\ Scheinerstr. 1, D-81679, Munich, Germany \\ email: burkert@usm.lmu.de
}

\begin{abstract}
Detailed models of galactic disk formation and evolution require knowledge about the initial conditions under which disk galaxies form, the boundary conditions that affect their secular evolution and the microphysical processes that drive the multi-phase interstellar medium and regulate star formation. Unfortunately, up to now, most of these ingredients are still poorly understood. The challenge therefore is to still construct realistic models of galactic disks with predictive power.
\end{abstract}

Keywords. Galaxy: disk, galaxies: formation, galaxies: evolution, ISM: general, ISM: clouds, hydrodynamics, turbulence

\section{Initial and Boundary Conditions: The Cosmological Angular Momentum Problem}

The radial surface density profiles of galactic disks are determined by the gravitational potential of the galaxy that is dominated in the outer parts by dark matter and the specific angular momentum distribution of the infalling gas that dissipates its potential and kinetic energy while settling into centrifugal equilibrium in the inner regions of a dark matter halo. In addition one has to consider the secular evolution of galactic disks. Viscous angular momentum redistribution and selective gas loss in galactic winds strongly affects the evolution of disks making it difficult to infer the initial conditions from their presently observed structure.

Angular momentum is an important ingredient in order for galactic disks to form. It is generally assumed that, before collapse, gas and dark matter are well mixed and therefore acquire a similar specific angular momentum distribution (Peebles 1969; Fall \& Efstathiou 1980; White 1984). If angular momentum would be conserved during gas infall, the resulting disk size should be directly related to the specific angular momentum $\lambda^{\prime}$ of the surrounding dark halo where (Bullock et al. 2001)

$$
\lambda^{\prime}=\frac{J}{\sqrt{2} M_{v i r} V_{v i r} R_{v i r}}
$$

with $R_{v i r}$ and $V_{v i r}^{2}=G M_{v i r} / R_{v i r}$ the virial radius and virial velocity of the halo, respectively, and $M_{v i r}$ its virial mass. Adopting a flat rotation curve, the disk scale length is (Mo et al. 1998; Burkert \& D'Onghia 04)

$$
R_{\text {disk }} \approx 8\left(\frac{\lambda^{\prime}}{0.035}\right)\left(\frac{v_{\max }}{200 k m / s}\right) k p c
$$

where $v_{\max }$ is the maximum rotational velocity in the disk.

Figure 1 shows the correlation between the disk scale length $R_{d i s k}$ and the maximum rotational velocity $v_{\max }$ for massive spiral galaxies (Courteau 1997) which is consistent with a mean value of $\lambda^{\prime} \approx 0.025$. This value is somewhat smaller than the theoretically 


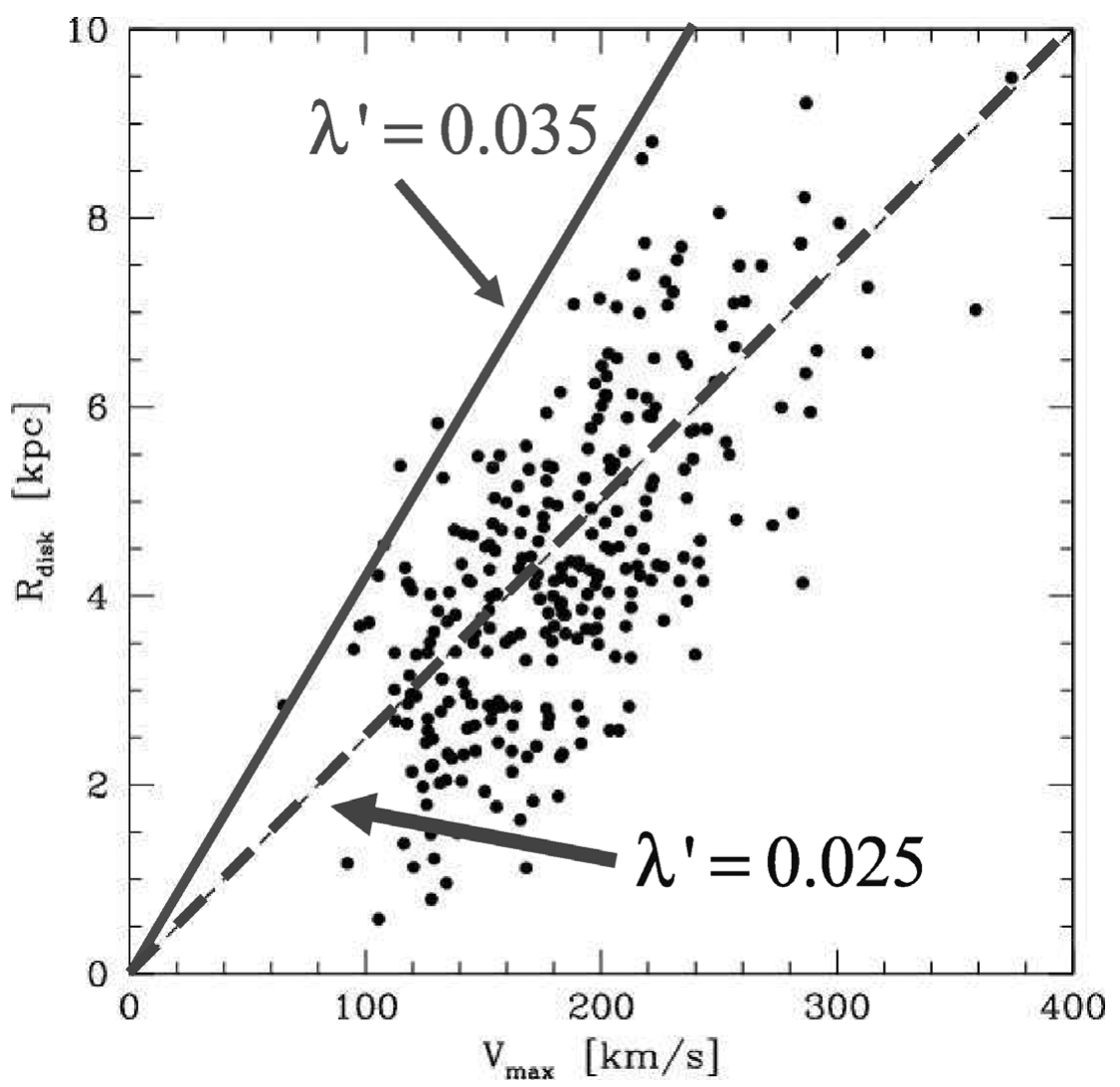

Figure 1. The observed scale lengths versus the maximum rotational velocities of galactic disks are shown for the Courteau (1997) sample. The sold line shows the theoretically predicted correlation for $\lambda^{\prime}=0.035$. The dashed curve corresponds to $\lambda^{\prime}=0.025$.

predicted value of $\lambda^{\prime}=0.035$, indicating that the gas could on average have lost some amount of angular momentum during the phase of decoupling from the dark component and settling into the equatorial plane.

This result is promising. The situation is however far less satisfactory when we consider more detailed numerical models of gas infall and accumulation in galactic disks. Many simulations of galactic disk formation suffer from catastrophic angular momentum loss which leads to disks with unreasonably small scale lengths and surface densities that are too large. The origin of this problem has been attributed to a strong clumping of the infalling gas which looses angular momentum by dynamical friction within the surrounding dark matter halo (Navarro \& Benz 1991, Navarro \& Steinmetz 2000). Other possibilites are low numerical resolution (Governato et al. 2004, 2007), the effect of substantial and major mergers (d'Onghia et al. 2006) or artificial secular angular momentum transfer from the cold disk to its hot surrounding (Okamoto et al. 2003). Over the years many groups have tried to solve this problem by including star formation and energetic feedback (e.g. Sommer-Larsen et al. 2003, Abadi et al. 2003, Springel \& Hernquist 2003, Robertson et al. 2004, Oppenheimer \& Dave 2006, Dubois \& Teyssier 2008). The results are however confusing. First of all the origin of the angular momentum problem is not clearly understood. Secondly, no reasonable, universally applicable feedback prescription has been found that would lead to the formation of large-sized, late-type disks, not only for one special case, but in general. 
Progress in our understanding of the cosmological angular momentum problem has recently been achieved by Zavala et al. (2008) who confirmed that the specific angular momentum distribution of the disk forming material follows closely the angular momentum evolution of the dark matter halo. The dark matter angular momentum grows at early times as a result of large-scale tidal torques, consistent with the prediction of linear theory and remains constant after the epoch of maximum expansion. During this late phase however angular momentum is redistributed within the dark halo with the inner dark halo regions loosing up to $90 \%$ of their specific angular momentum to the outer parts. The process leading to this angular momentum redistribution is not discussed in details. It is however likely that substantial mergers with mass ratios less than 10:1 that are expected to occur frequently even at late phases during galaxy formation perturb the halo and generate global asymmetries in the mass distribution that are known to be an efficient mechanism for angular momentum transfer. Small satellite infall is probably of minor importance. It would be interesting to study the role of major and minor mergers in this process in greater details.

It is then likely that any gas residing in the inner regions during such an angular momentum redistribution phase will also loose most of its angular momentum, independent of whether the gas resides already in a protodisk, is still confined to dark matter substructures or is in an extended, diffuse distribution. Zavala et al. (2008) (see also Okamoto et al., 2005 and Scannapieco et al., 2008) show that efficient heating of the gas component can prevent angular momentum loss, probably because most of the gaseous component resides in the outer parts of the dark halo during its angular momentum redistribution phase. The gas would then actually gain angular momentum rather than loose it and could lateron settle smoothly into an extended galactic disk in an ELS-like (Eggen, Lynden-Bell \& Sandage 1962) accretion phase.

Unfortunately little is known about the energetic processes that could lead to such an evolution. Obviously, star formation must be delayed during the protogalactic collapse phase in order for the gas to have enough time to settle into the plane before condensing into stars. However star formation is also required in order to heat the gas and preventing it from collapsing prior to the angular momentum redistribution phase. Scannapieco et al. (2008) show that their supernova feedback prescription is able to regulate star formation while at the same time pressurizing the gas. Their models are however still not efficient enough in order to produce disk-dominated, late-type galaxies. Large galactic disks are formed. The systems are however dominated by a central, massive, low-angular momentum stellar bulge component. This is in contradiction with observations which indicate a large fraction of massive disk galaxies with bulge-to disk ratios smaller than $50 \%$ (Weinzirl et al. 2008) that cannot be produced currently by numerical simulations of cosmological disk formation.

\section{Energetic Feedback and Star Formation}

As discussed in the previous section, star formation and energetic feedback plays a dominant role in understanding the origin and evolution of galactic disks and in determining the morphological type of disk galaxies. Scannapieco et al. (2008) for example demonstrate that the same initial conditions could produce either an elliptical or a disk galaxy, depending on the adopted efficiency of gas heating during the protogalactic collapse phase. We do not yet have a consistent model of the structure and evolution of the multi-phase, turbulent interstellar medium and its condensation into stars. This situation is now improving rapidly due to more sophisticated numerical methods and fast computational platforms that allow us to run high-resolution models, incorporating a 
large number of possibly relevant physical processes (Wada \& Norman 2002, Krumholz \& McKee 2005, Tasker \& Bryan 2008, Robertson \& Kravtsov 2008). Most cosmological simulations however have up to now adopted simplified observationally motivated descriptions of star formation that are based on the empirical Kennicutt relations (Kennicutt 1998, 2007) that come in two different versions. The first relation (K1) represents a correlation between the star formation rate per surface area $\Sigma_{S F R}$ and the gas surface density $\Sigma_{g}$, averaged over the whole galaxy

$$
\Sigma_{S F R}^{(K 1)}=2.5 \times 10^{-4}\left(\frac{\Sigma_{g}}{M_{\odot} / p c^{2}}\right)^{1.4} \frac{M_{\odot}}{k p c^{2} y r}
$$

The second relation (K2) includes a dependence on the typical orbital period $\tau_{\text {orb }}$ of the disk

$$
\Sigma_{S F R}^{(K 2)}=0.017\left(\frac{\Sigma_{g}}{M_{\odot} / p c^{2}}\right)\left(\frac{10^{8} y r s}{\tau_{o r b}}\right) \frac{M_{\odot}}{k p c^{2} y r} .
$$

These relationships have been derived from observations as an average over the whole disk. They are however often also used as theoretical prescriptions for the local star formation rate which appears observationally justified if the total gas surface densitiy $\Sigma_{g}$ is replaced by the local surface density of molecular gas. The origin of both relationships is not well understood yet. For example, Li et al. (2005, 2006) ran SPH simulations of a gravitationally unstable gaseous disks, confined by the gravitational potential of a surrounding dark matter halo. Gravitationally bound gas clumps form in their disks and are replaced by accreting sink particles. The authors assume that $30 \%$ of the mass of these particles is in stars with the rest remaining gaseous. However, no stellar feedback or a destruction mechanism of the partly gaseous sink particles is adopted. The star formation surface density is investigated for different galactic disk models with different rotational velocities and initial gas surface densities. The authors find a good agreement with the first Kennicutt relation (K1) if they correlate $\Sigma_{S F R}$ with $\Sigma_{g}$ at a time when the star formation rate has decreased by a factor of 2.7 with respect to the initial value which in their model typically corresponds to an evolutionary time of a few $10^{7}$ yrs. The significance of this result is however not clear. Obviously, the galaxies studied by Kennicutt are much older and in a phase of self-regulated star formation that cannot be considered in models without energetic feedback. In addition, the authors cannot reproduce the second relation (K2), indicating that $\mathrm{K} 2$ is not directly related to $\mathrm{K} 1$ but instead represents a second constraint for theoretical models.

We can combine $\mathrm{K} 1$ and $\mathrm{K} 2$ and derive a relationship between the average gas density in galactic disks and their orbital period

$$
\Sigma_{g} \sim \tau_{o r b}^{-2.5} \sim\left(\frac{v_{\text {rot }}}{R_{\text {disk }}}\right)^{2.5}
$$

where $v_{\text {rot }}$ and $R_{d i s k}$ are the rotational velocity and the size of the galactic disk, respectively. This result is puzzling as it is not clear why the kinematical properties of galactic disks should correlate with their gas surface densities especially in galaxies of Milky Way type or earlier where the gas fraction is small compared to the mass in stars. Recent detailed hydrodynamical simulations of disk galaxies by Robertson \& Kravtsov (2008), including low-temperature gas cooling and molecular hydrogen physics can indeed reproduce both Kennicutt relations. The authors however note themselves that the physical reason for the origin of the K2-relation in their simulations is unclear. They argue that in disk galaxies with exponential density profiles the disk surface density should scale with 


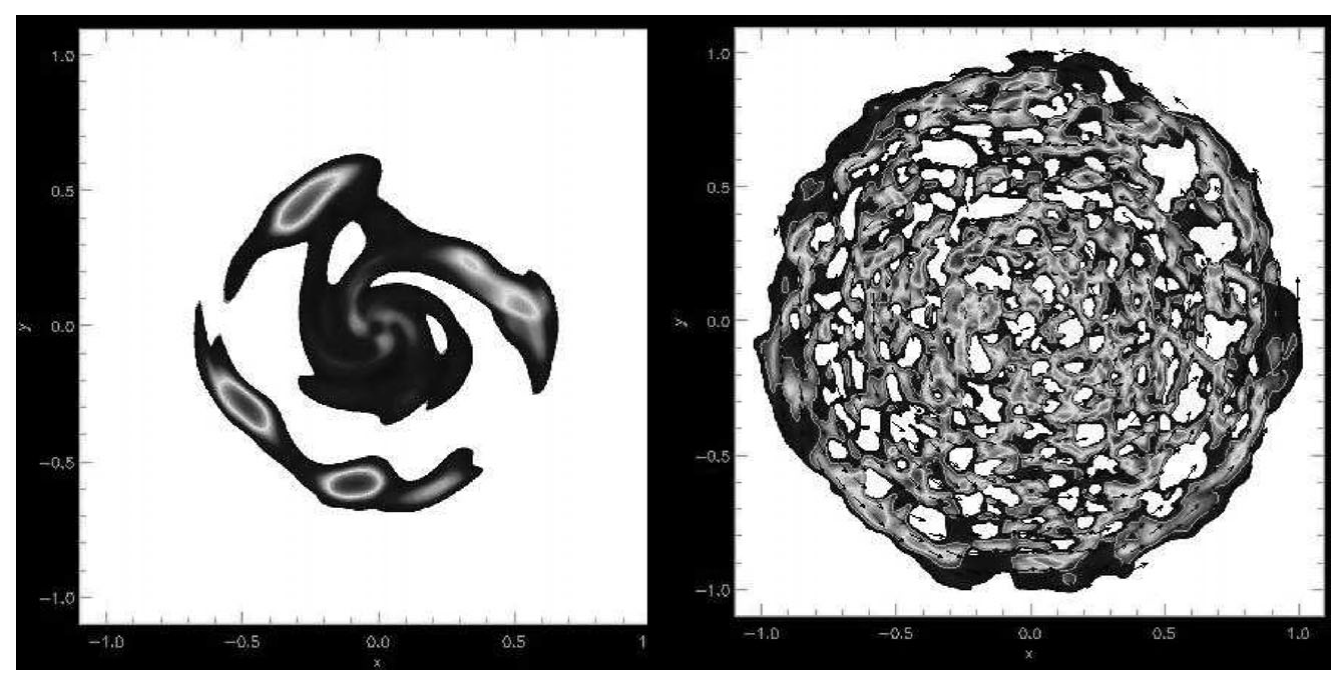

Figure 2. Gas surface density of an initially homogeneous, gravitationally unstable gas-rich galactic disk, embedded in a dark matter halo. The left panel shows the gas density distribution if star formation is suppressed. The disk forms a few massive gaseous clumps that spiral into the center by dynamical friction. The situation is however very different if star formation and stellar energy feedback is included. In this case, supernova explosions efficiently disrupt dense clumps before they can merge into giant cloud complexes while at the same time generating a highly turbulent and filamentary multi-phase interstellar medium (Burkert et al. 2009).

the orbital period as $\Sigma_{d} \sim \tau_{\text {orb }}^{-2}$. In this case, K2 requires that $\Sigma_{g} \sim \Sigma_{d}^{1.2} \sim\left(\Sigma_{*}+\Sigma_{g}\right)^{1.2}$ with $\Sigma_{*}$ the stellar surface density. It is not clear why this relation should hold, especially for disks with $\Sigma_{*}>\Sigma_{g}$.

\section{Secular Evolution and Turbulence in Galactic Disks}

Bullock et al. (2001) demonstrated that dark halos have a universal angular momentum distribution that should also be characteristic for the infalling gas component. Van den Bosch et al. (2001) lateron showed that this angular momentum distribution is not consistent with the observed distribution of exponential galactic disks indicating that viscous angular momentum redistribution in galactic disks must have played an important role. The viscosity is likely driven by interstellar turbulence which is a result of stellar energetic feedback processes (see Fig. 2) or global disk instabilities (magneto-rotational instability or gravitational instability). Note, that viscous effects will make the angular momentum problem worse as viscosity in general removes angular momentum from the dominant mass component in the disk.

The viscous formation of exponential stellar disks from gas disks with various different surface density distributions has been studied e.g. by Slyz et al. (2002). Their numerical simulations show that exponential disks form if the star formation timescale is of order the viscous timescale. Genzel et al. (2008) derived a timescale for turbulent viscosity in galactic disks of

$$
\tau_{v i s c}=\frac{1}{\alpha}\left(\frac{v_{r o t}}{\sigma}\right)^{2} \tau_{o r b}
$$

where $\alpha$ is of order unity. $\tau_{v i s c} \approx 10^{10}$ yrs for disks like the Milky Way with $\sigma \approx 10$ $20 \mathrm{~km} / \mathrm{s}$ and self-regulated low star formation rates. $\mathrm{H} \alpha$ integral field spectroscopy has 
however detected $z \sim 2$ star forming disk galaxies with large random gas motions of order $40 \mathrm{~km} / \mathrm{s}$ to $60 \mathrm{~km} / \mathrm{s}$ and viscous timescales of less than $10^{9} \mathrm{yrs}$ (Genzel et al., 2006, 2008, Förster-Schreiber et al. 2006). Interestingly, for these objects, the star formation timescales are again similar to the viscous timescales, leading to star formation rates of $100 \mathrm{M}_{\odot} / \mathrm{yr}$ and confirming that galactic disk gas turbulence, star formation and secular evolution are intimately coupled. The origin of the clumpiness and high turbulence in redshift 2 disks is not well understood yet. It seems likely that it is a result of substantial filamentary gas inflow (Dekel et al. 2008), combined with gravitational instabilites in the disk (Bournaud et al. 2007).

Turbulence seems to regulate star formation not only on large galactic scales but also on local cloud scales. Most of the molecular gas in the Milky Way is found in giant molecular clouds with masses of order $10^{4}-10^{6} M_{\odot}$, temperatures of order $10 \mathrm{~K}$ and average densities of order $100 \mathrm{~cm}^{-3}$. As their Jeans mass is of order $20 \mathrm{M}_{\odot}$ which is much smaller than their total mass one would expect that molecular clouds should collapse and condense into stars on a local free-fall time which is of order $5 \times 10^{6}$ yrs. Adopting a total molecular mass of $M_{H_{2}} \approx 3 \times 10^{9} M_{\odot}$ and assuming that a fraction $\eta_{S F} \approx 0.1$ of the molecular cloud's mass forms stars, the inferred mean star formation rate in the Milky Way is

$$
S F R=\eta_{S F} \frac{M_{H_{2}}}{\tau_{f f}} \approx 60 M_{\odot} / y r
$$

which is an order of magnitude larger than observed. A possible solution of this problem is turbulence. Molecular clouds are observed to be driven and shaped by supersonic turbulence that might strongly affect their stability and star formation rate. The origin of this turbulent motion and its impact on the cloud's lifetime and star formation process is not well understood yet. It is however likely that large-scale disk turbulence is the seed for turbulence in molecular clouds which again affects the star formation rate that in turn drives again large scale disk turbulence and by this also the viscous secular evolution of galactic disks.

\section{Summary}

We are currently living in a very exciting time where the various complex processes that can affect galactic disk formation and evolution are being uncovered and studied observationally and theoretically. Combined with the now well established cold dark matter structure formation scenario the time seems ripe for self-consistent models of galaxy formation with predictive power. Given the high capacities of present-day supercomputers it is understandable that one tries to including as many processes as possible, most of which being however not well understood. These models not only suffer from a large number of free parameters. They also do not necessarily lead to insight as they are so complex and depend on so many different implemented physical aspects that it is impossible to clearly understand what in the end the origin of a certain result will be.

I wonder whether one needs a high complexity in order to understand important questions of galactic disk evolution, like the two KS laws, the origin of turbulence in the diffuse interstellar medium or in molecular clouds or the origin of the strong correlation between the viscous timescale and the star formation timescale.

Let us try to solve simple questions first before we focus on the complex puzzles that involve many processes that are not well understood yet. 


\section{References}

Abadi, M. G., Navarro, J. F., Steinmetz, M., \& Eke, V. R. 2003, ApJ, 591, 499

Bournaud, F., Elmegreen, B. G., \& Elmegreen, D. M. 2007, ApJ, 670, 237

Bullock, J. S., Dekel, A., Kolatt, T. S., Kravtsov, A. V., Klypin, A. A., Porciani, C., \& Primack, J.R. 2001, ApJ, 555, 240

Burkert, A. \& d'Onghia, E. 2004, in Penetrating Bars through Masks of Cosmic Dust: The Hubble Tuning Fork Strikes a New Note, eds. D.L. Block, I. Puerari, K.C. Freeman, R. Groess and E.K. Block, ASSL 319, 341

Courteau, S. 1997, AJ, 114, 2402

Dekel, A. et al. 2008, Nature, in press, arXiv:0808.0553

Dubois, Y. \& Teyssier, R. 2008, AA, 477, 79

D'Onghia, E., Burkert, A., Murante, G., \& Khochfar, S. 2006, MNRAS, 372, 1525

Eggen, O. J., Lynden-Bell, D., \& Sandage, A. R. 1962, ApJ, 136, 748

Fall, S. M. \& Efsthatiou, G. 1980, MNRAS, 193, 189

Förster-Schreiber, N. M. et al. 2006, ApJ, 645, 1062

Genzel, R. et al. 2006, Nature, 442, 786

Genzel, R. et al. 2008, Apj, submitted, arXiv:0807.1184

Governato, F. et al. 2004, ApJ, 607, 688

Governato, F. et al. 2007, MNRAS, 374, 1479

Kennicutt, R. C., Jr. 1998, ApJ, 498, 541

Kennicutt, R. C., Jr. et al. 2007, ApJ, 671, 333

Krumholz, M. R. \& McKee, C. F. 2005, ApJ, 630, 250

Li, Y., Mac Low, M.-M., \& Klessen, R. S. 2005, ApJ, 620, L19

Li, Y., Mac Low, M.-M., \& Klessen, R. S. 2005, ApJ, 639, 879

Mo, H. J., Mao, S., \& White, S. D. M. 1998, MNRAS, 295, 319

Navarro, J. \& Benz, W. 1991, ApJ, 380, 320

Navarro, J. \& Steinmetz, M. 2000, ApJ, 538, 477

Okamoto, T., Jenkins, A., Eke, V. R., Quilis, V., \& Frenk, C. S. 2003, MNRAS, 345, 429

Okamoto, T., Eke, V. R., Frenk, C. S., \& Jenkins, A. 2005, MNRAS, 363, 1299

Oppenheimer, B. D. \& Dave, R. 2006, MNRAS, 373, 1265

Peebles, P. J. E. 1969, ApJ, 155, 393

Robertson, B., Yoshida, N., Springel, V., \& Hernquist, L. 2004, ApJ, 606, 32

Robertson, B. \& Kravtsov, A. V. 2008, ApJ, 680, 1083

Scannapieco, C., Tissera, P. B., White, S. D. M., \& Springel, V. 2008, MNRAS, 389, 1137

Slyz, A. D., Devriendt, J. E. G., Silk, J., \& Burkert,A. 2002, MNRAS, 333, 894

Sommer-Larsen, J., Götz, M., \& Portinari, L. 2003, ApJ, 596, 47

Springel, V. \& Hernquist, L. 2003, MNRAS, 339, 289

Tasker, E. J. \& Bryan, G. L. 2008, ApJ, 673, 810

Van den Bosch, F. C., Burkert, A., \& Swaters, R. A. 2001, MNRAS, 326, 1205

Wada, K. \& Norman, C. A. 2007, ApJ, 660, 276

Weinzirl, T., Jogee, S., Khochfar, S., Burkert, A., \& Kormendy, J. 2008, ApJ, submitted (arXiv:0807.0040)

White, S. D. M. 1984, MNRAS, 286, 38

Zavala, J., Okamoto, T., \& Frenk, C. S. 2008, MNRAS, 387, 839 


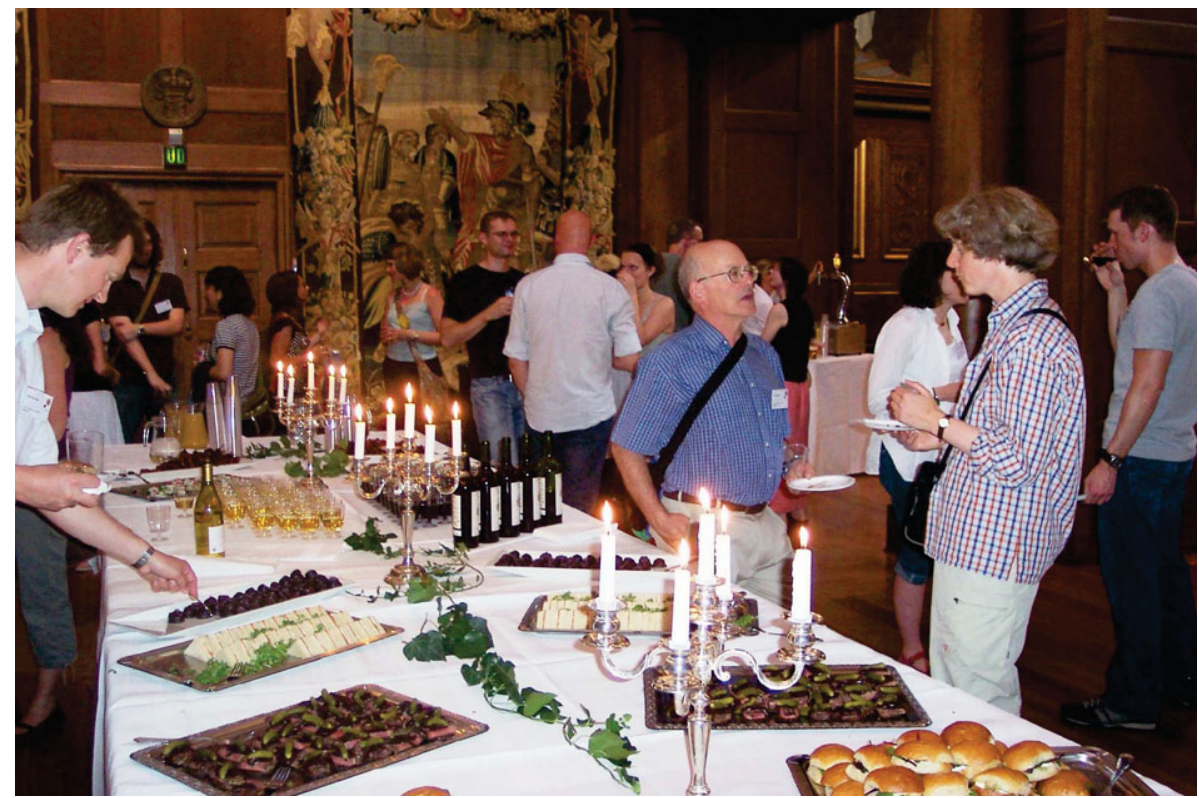

Starting the meeting: The welcome reception at Copenhagen University. Frank Grundahl, Ken Freeman and Sofia Feltzing in the foreground.

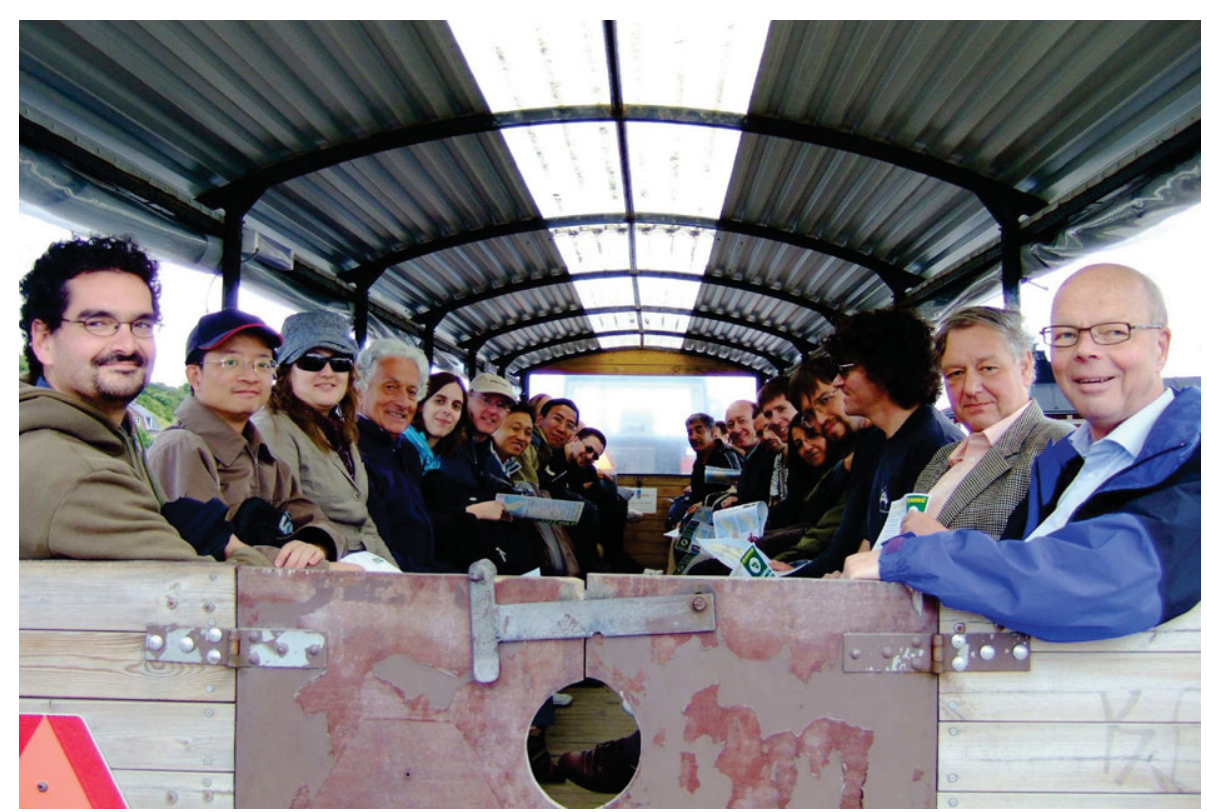

- and ending it: The Saturday excursion on the way to Uranienborg in one of the characteristic tractor-powered buses on Hven. 